\title{
Realizing $n$-Type Field-Effect Performance via Introducing Trifluoromethyl Groups into the Donor-Acceptor Copolymer Backbone
}

Congyuan Wei, ${ }^{\dagger} \S$ Weifeng Zhang, ${ }^{\dagger}$ Jianyao Huang, ${ }^{\dagger}$ Hao Li, ${ }^{\dagger}$ Yankai Zhou, ${ }^{\dagger}$ and Gui $\mathrm{Yu}^{*, \dagger, \S}$

${ }^{\dagger}$ Beijing National Laboratory for Molecular Sciences, CAS Research/Education Center for Excellence in Molecular Sciences, Institute of Chemistry, Chinese Academy of Sciences, Beijing 100190, P. R. China.

$\$$ School of Chemical Sciences, University of Chinese Academy of Sciences, Beijing 100049, P. R. China.

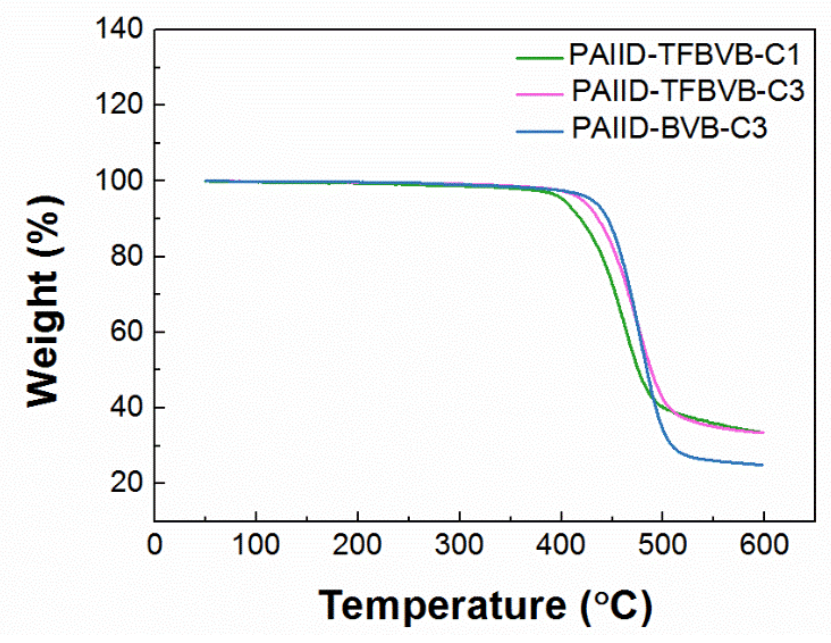

Figure S1. Thermogravimetric analysis (TGA) traces of PAIID-TFBVB-C1, PAIID-TFBVB-C3, and PAIID-BVB-C3. 

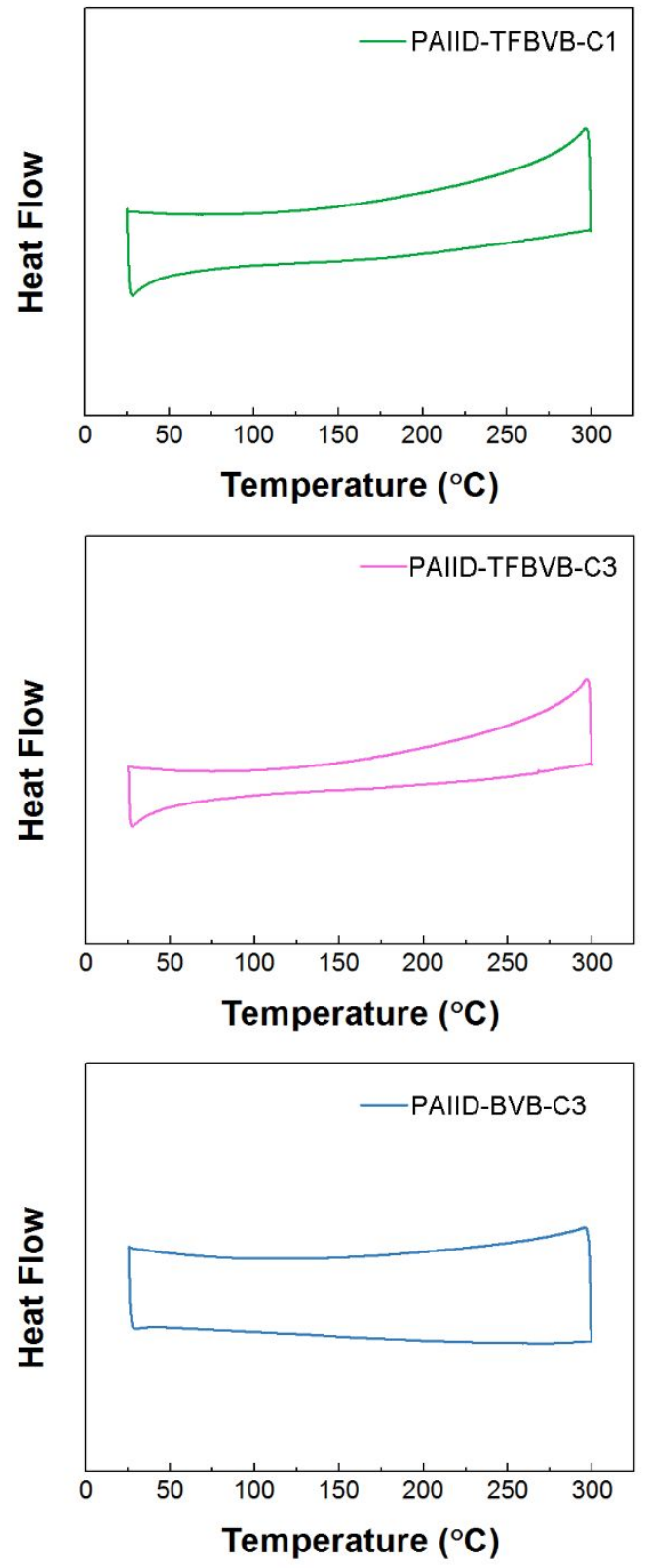

Figure S2. Differential scanning calorimetry (DSC) curves of PAIID-TFBVB-C1, PAIID-TFBVB-C3, and PAIID-BVB-C3. 


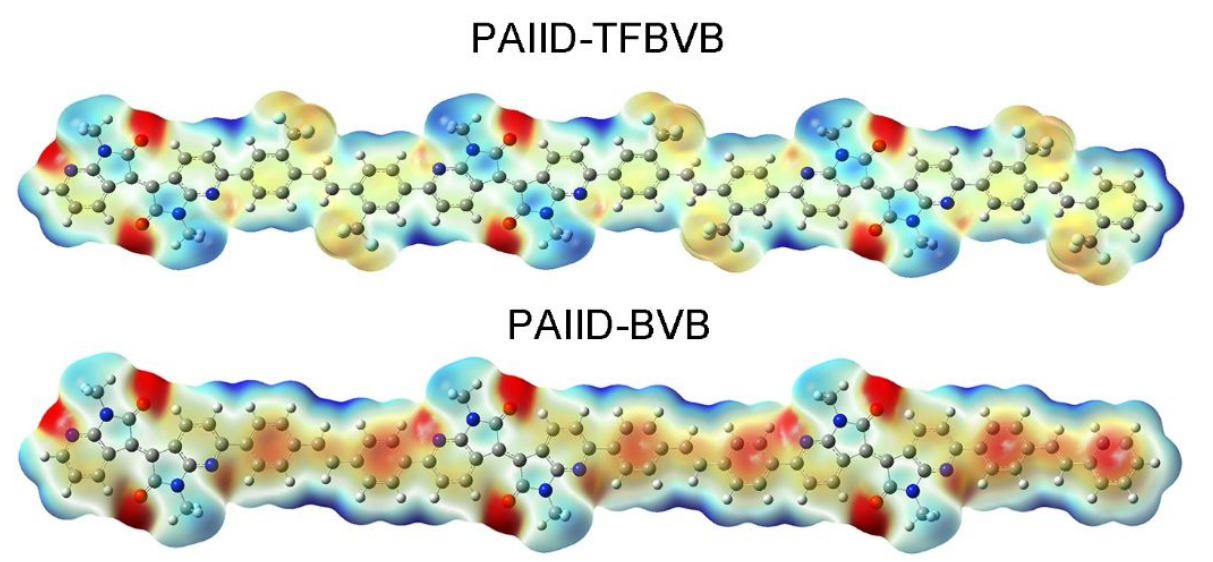

Figure S3. The electrostatic potential maps of the model molecules PAIID-TFBVB and PAIID-BVB.
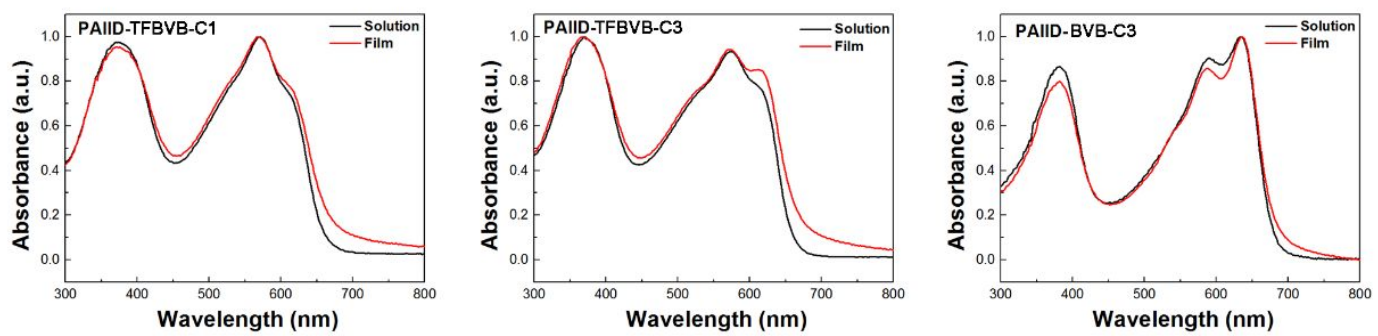

Figure S4. UV-vis absorption spectra of PAIID-TFBVB-C1, PAIID-TFBVB-C3, and PAIID-BVB-C3 in dilute chloroform solution and thin film.
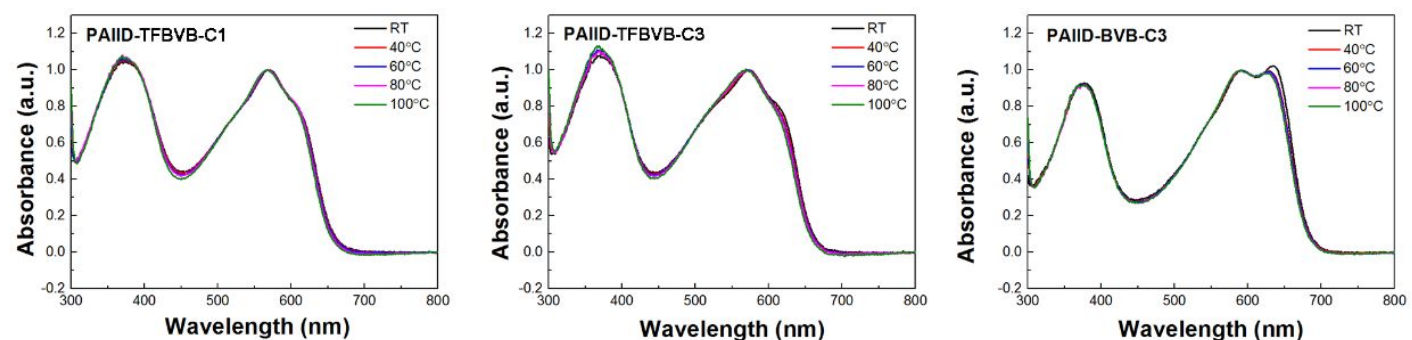

Figure S5. Temperature-dependent UV-vis absorption spectra of PAIID-TFBVB-C1,

PAIID-TFBVB-C3, and PAIID-BVB-C3 in dilute 1,2-dichlorobenzene solution. 

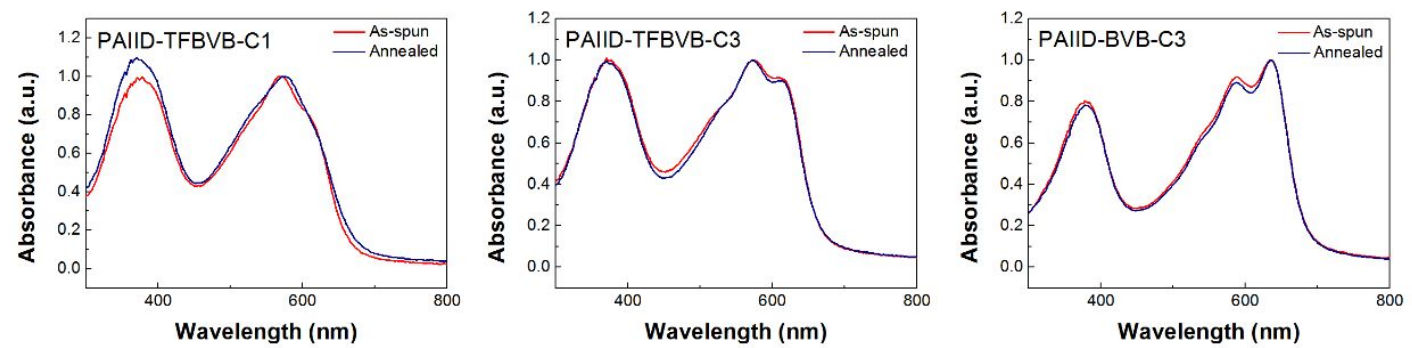

Figure S6. UV-vis absorption spectra of PAIID-TFBVB-C1, PAIID-TFBVB-C3 and PAIID-BVB-C3 thin film deposited from 1,2-dichlorobenzene solution before and after thermal annealing treatment $\left(280^{\circ} \mathrm{C}\right.$ for PAIID-TFBVB-C1 and PAIID-TFBVB-C3, $140^{\circ} \mathrm{C}$ for PAIID-BVB-C3).
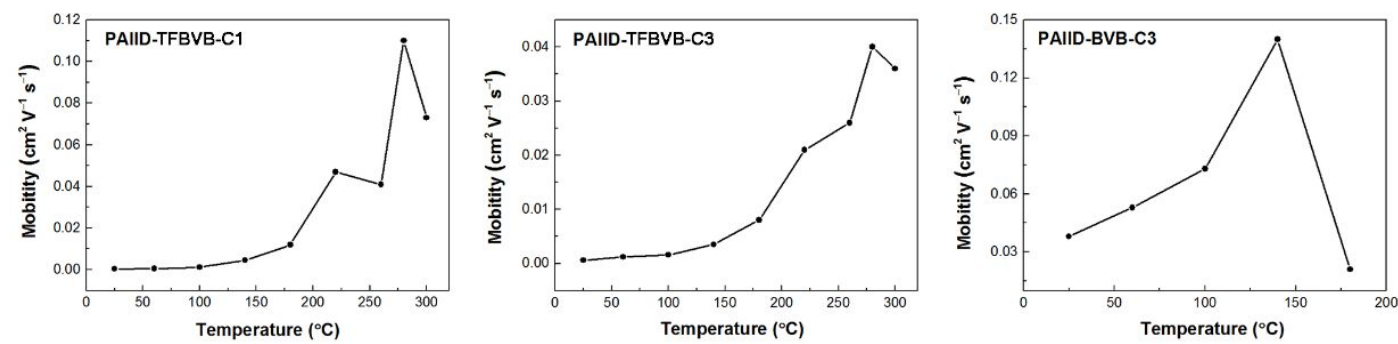

Figure S7. The annealing temperature-dependent mobilities of OFET devices based on PAIID-TFBVB-C1, PAIID-TFBVB-C3, and PAIID-BVB-C3. 

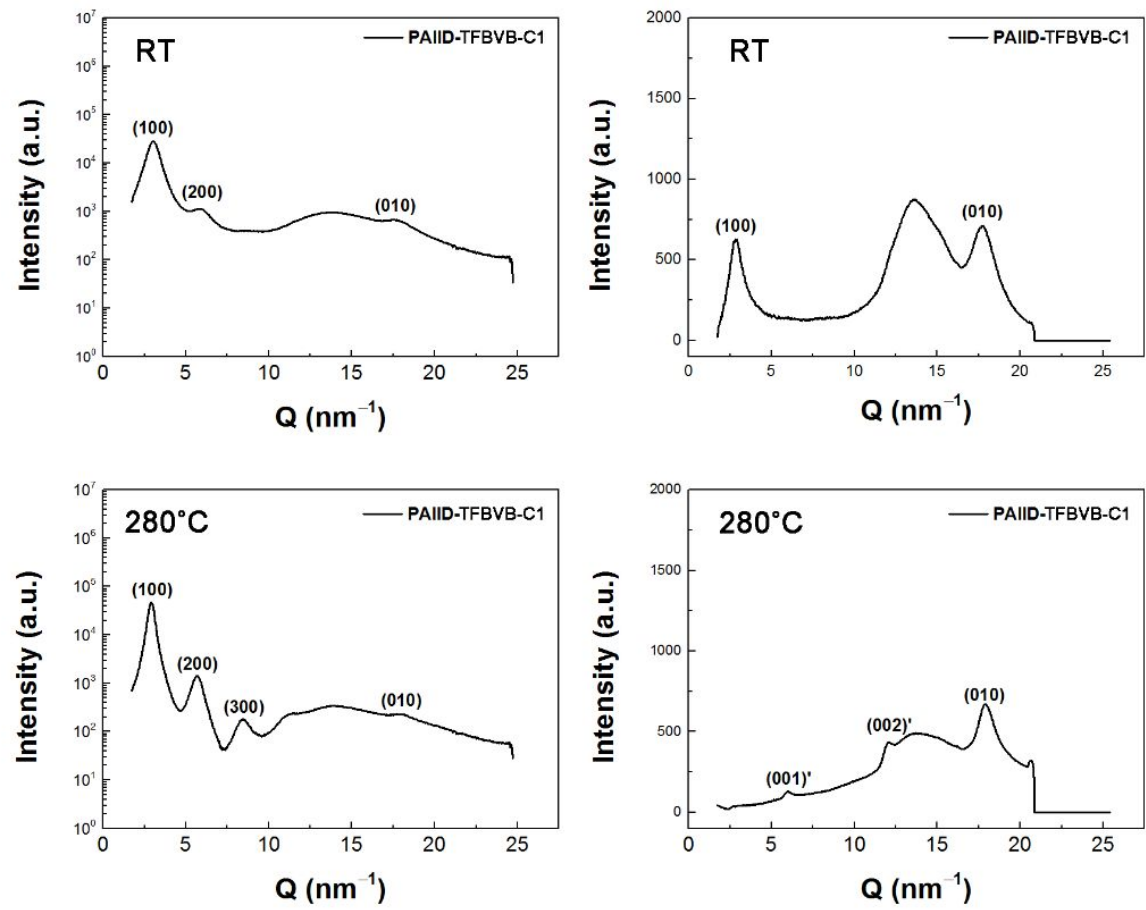

Figure S8. The out-of-plane (left) and in-plane (right) profiles of GIXRD patterns of PAIID-TFBVB-C1 thin film before and after annealing treatment.
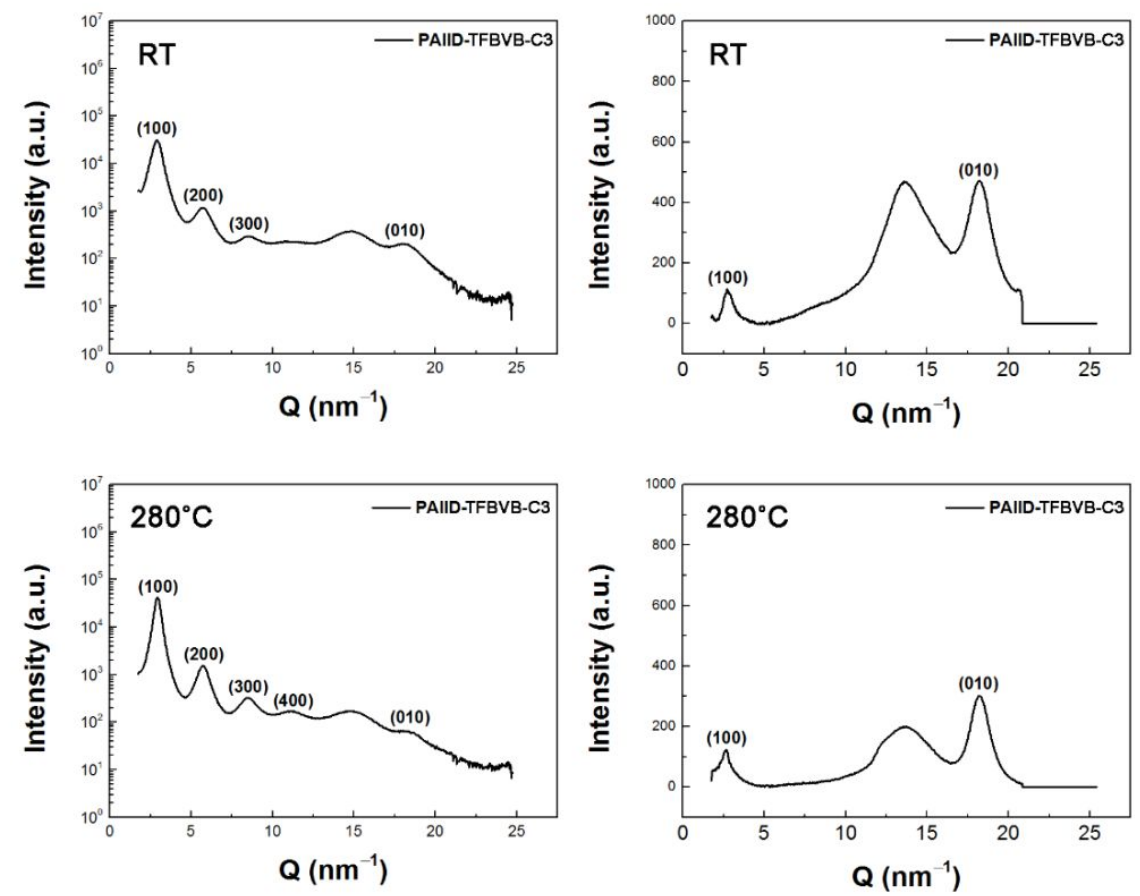

Figure S9. The out-of-plane (left) and in-plane (right) profiles of GIXRD patterns of PAIID-TFBVB-C3 thin film before and after annealing treatment. 

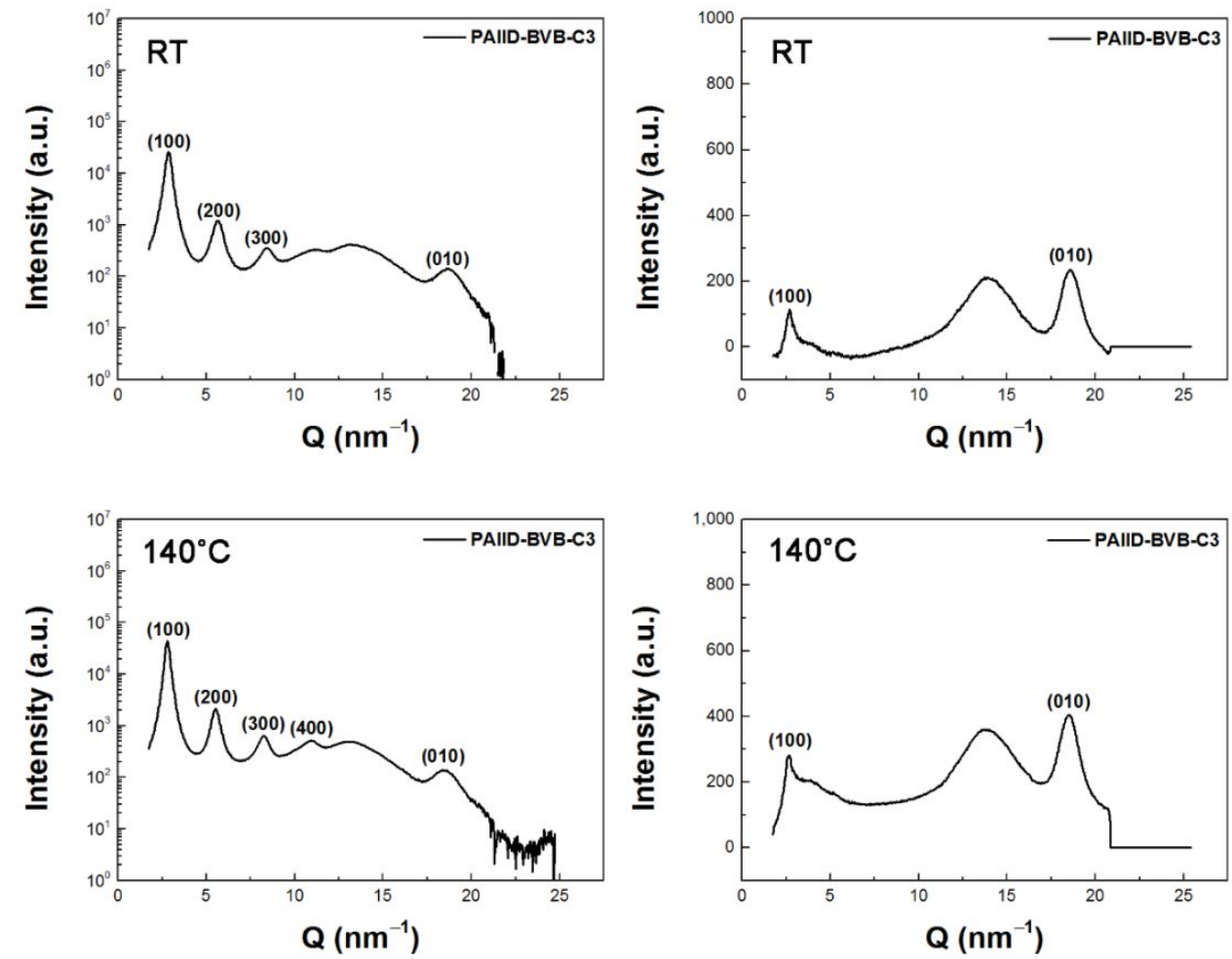

Figure S10. The out-of-plane (left) and in-plane (right) profiles of GIXRD patterns of PAIID-BVB-C3 thin film before and after annealing treatment.
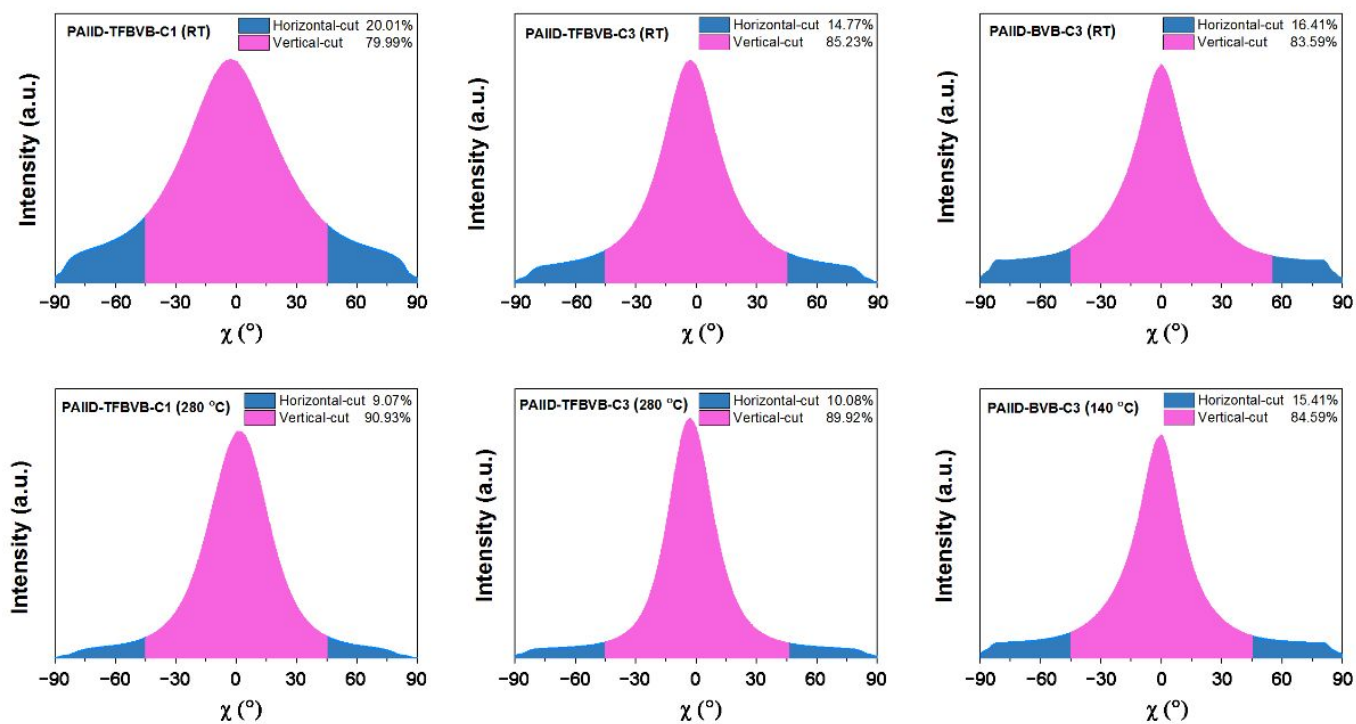

Figure S11. Pole figures of the (100) peak of PAIID-TFBVB-C1, PAIID-TFBVB-C3, and PAIID-BVB-C3 thin films before and after annealing treatment. 

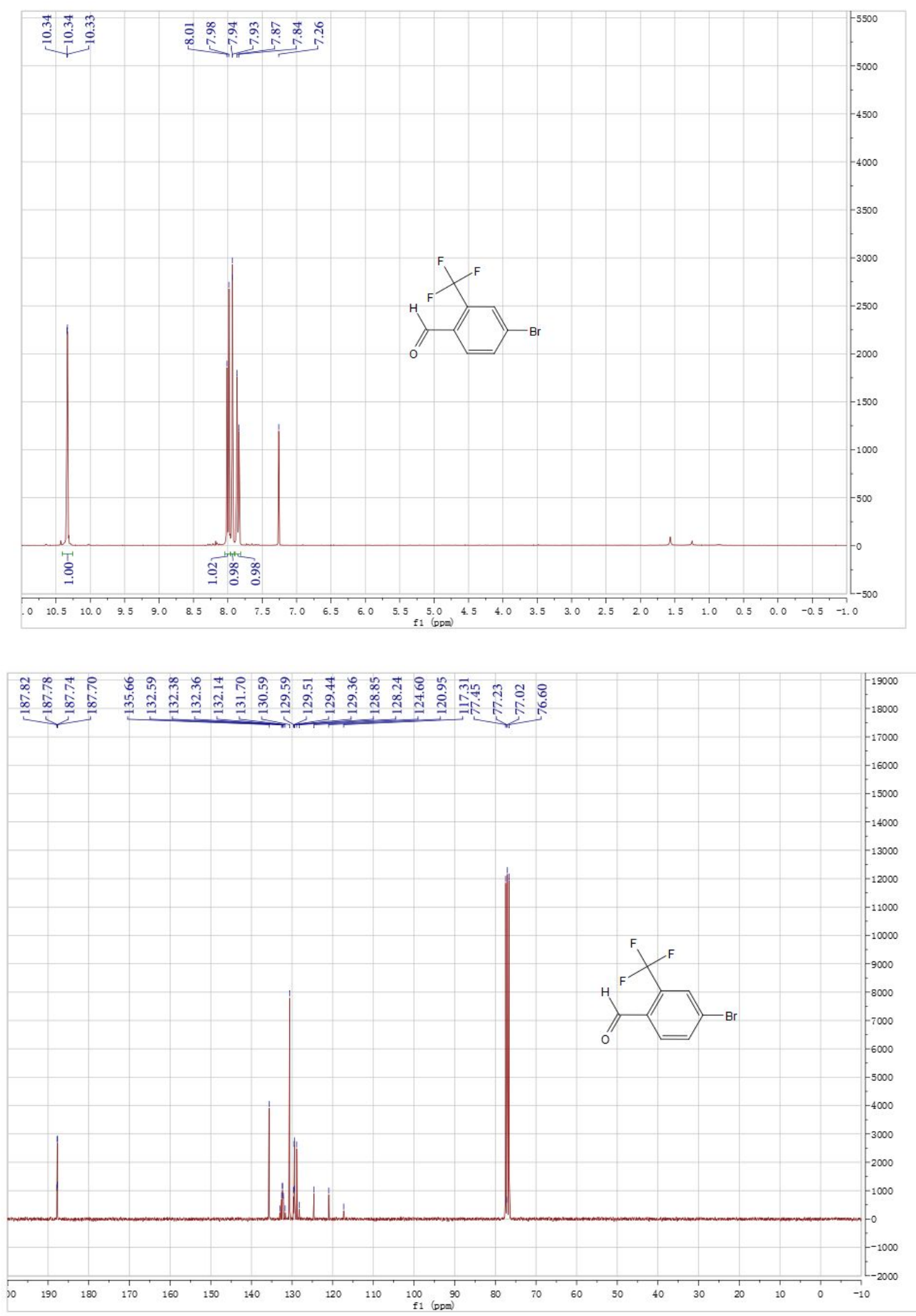

Figure S12. ${ }^{1} \mathrm{H}$ NMR and ${ }^{13} \mathrm{C}$ NMR spectra of compound 2. 

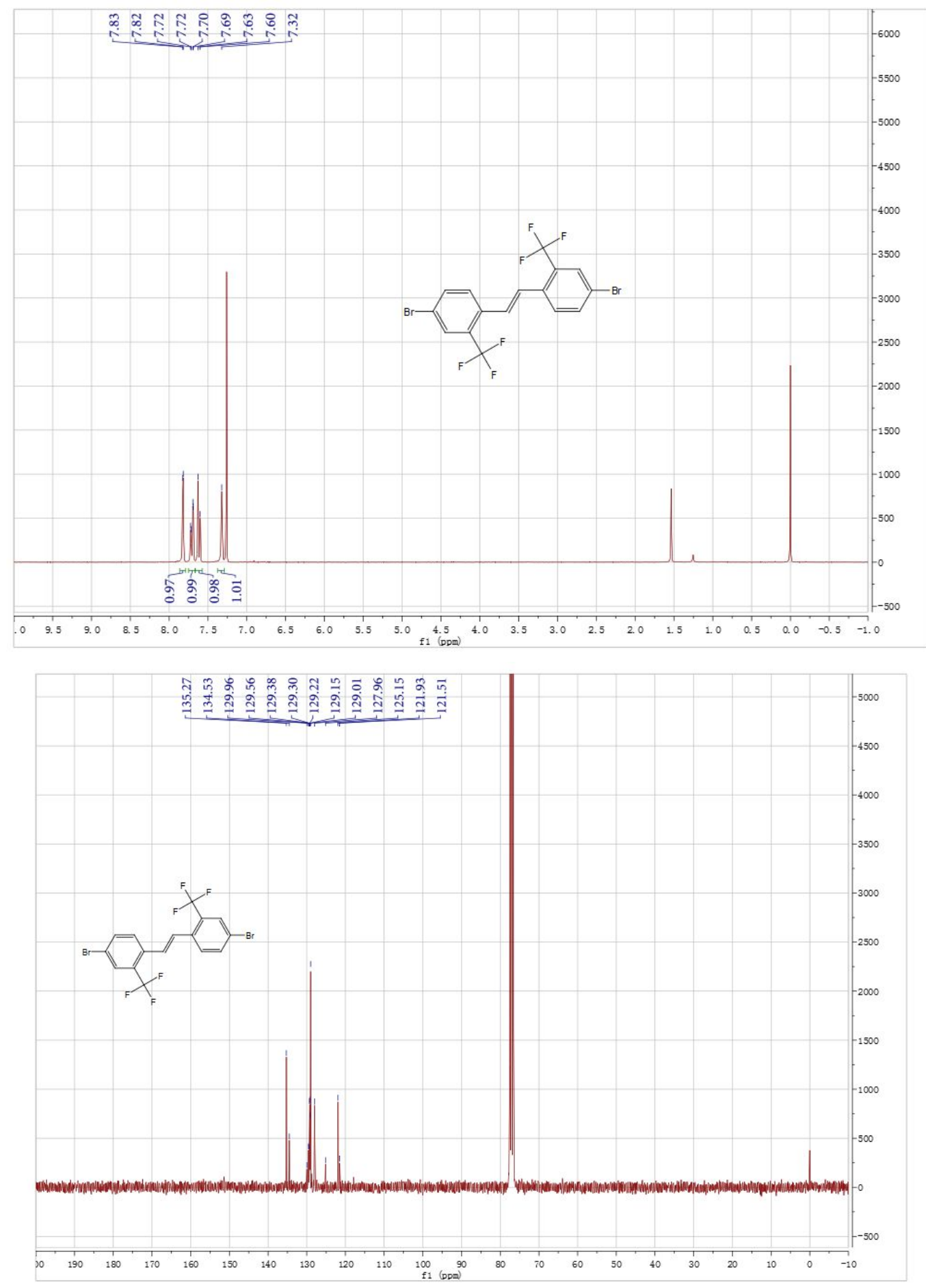

Figure S13. ${ }^{1} \mathrm{H}$ NMR and ${ }^{13} \mathrm{C}$ NMR spectra of compound 3. 

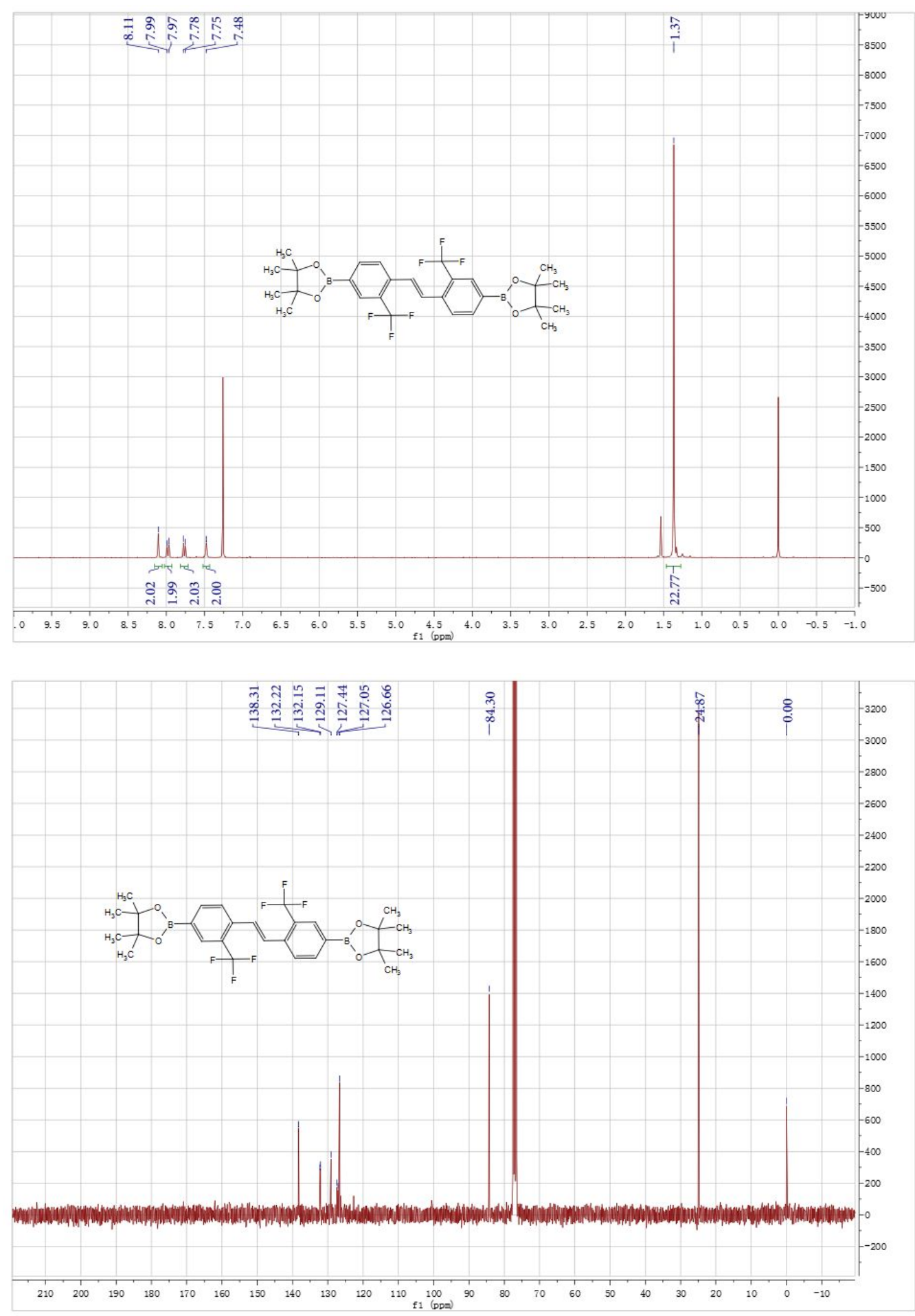

Figure S14. ${ }^{1} \mathrm{H}$ NMR and ${ }^{13} \mathrm{C}$ NMR spectra of compound 4. 

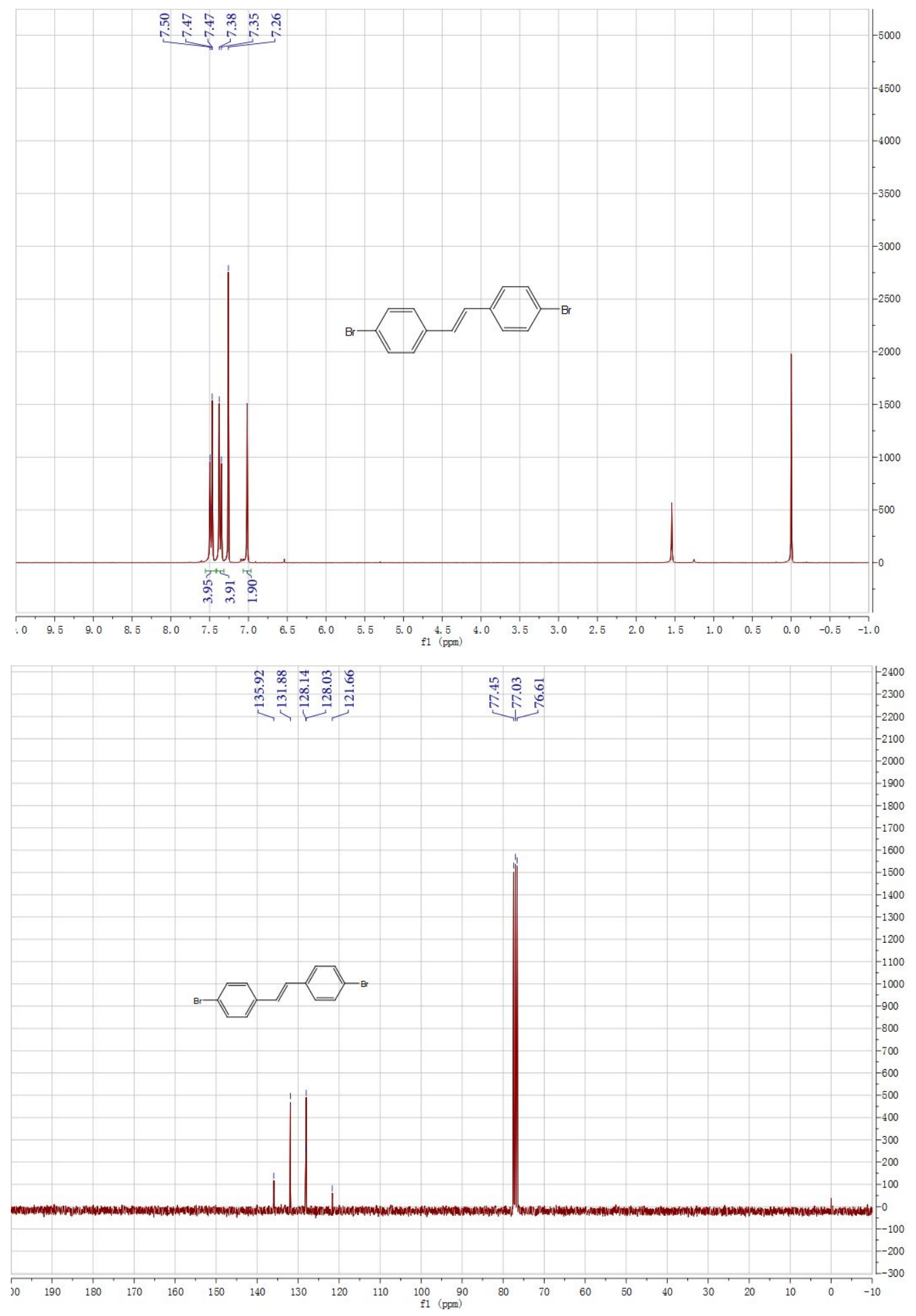

Figure S15. ${ }^{1} \mathrm{H}$ NMR and ${ }^{13} \mathrm{C}$ NMR spectra of compound 6. 

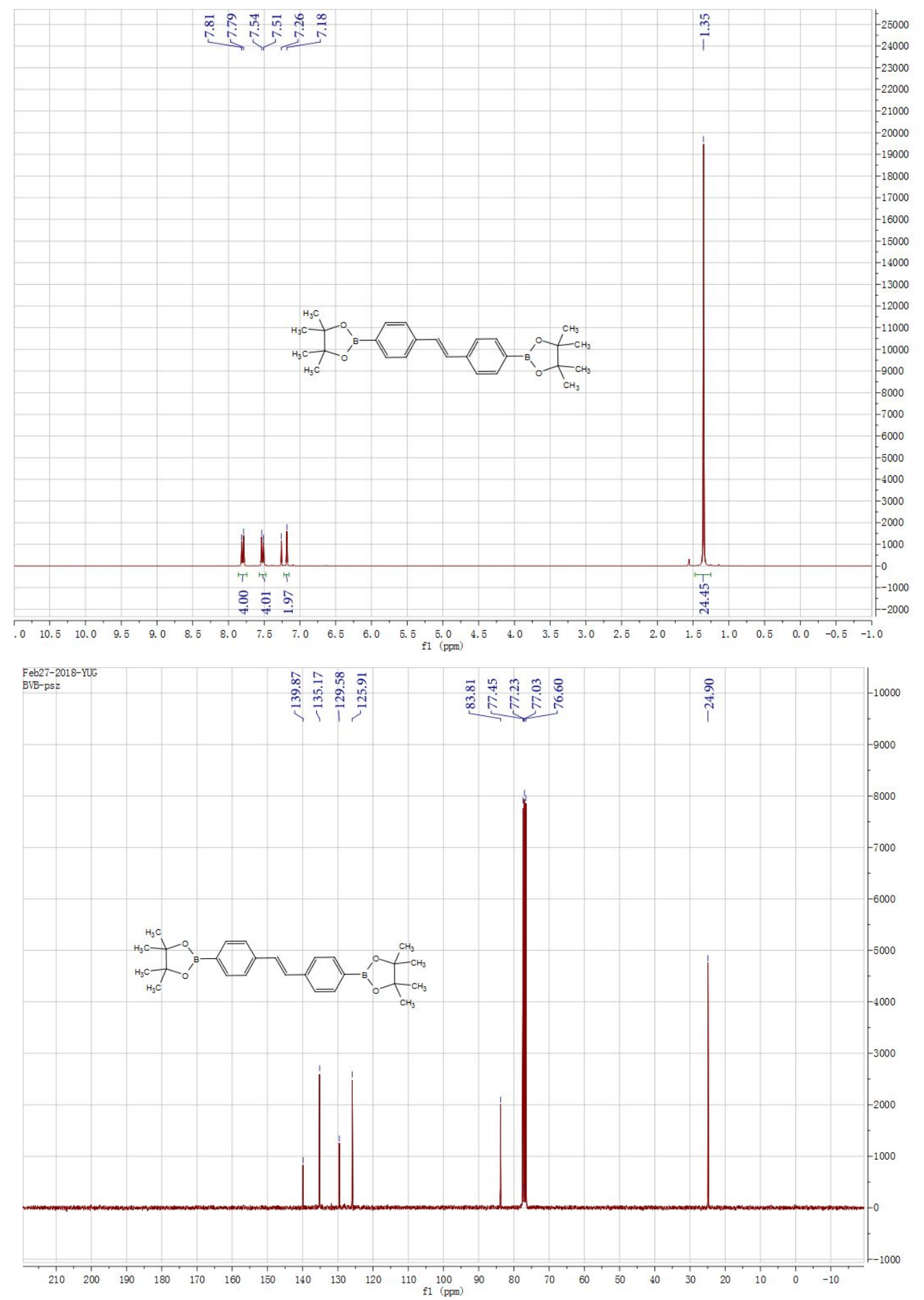

Figure S16. ${ }^{1} \mathrm{H}$ NMR and ${ }^{13} \mathrm{C}$ NMR spectra of compound 7. 


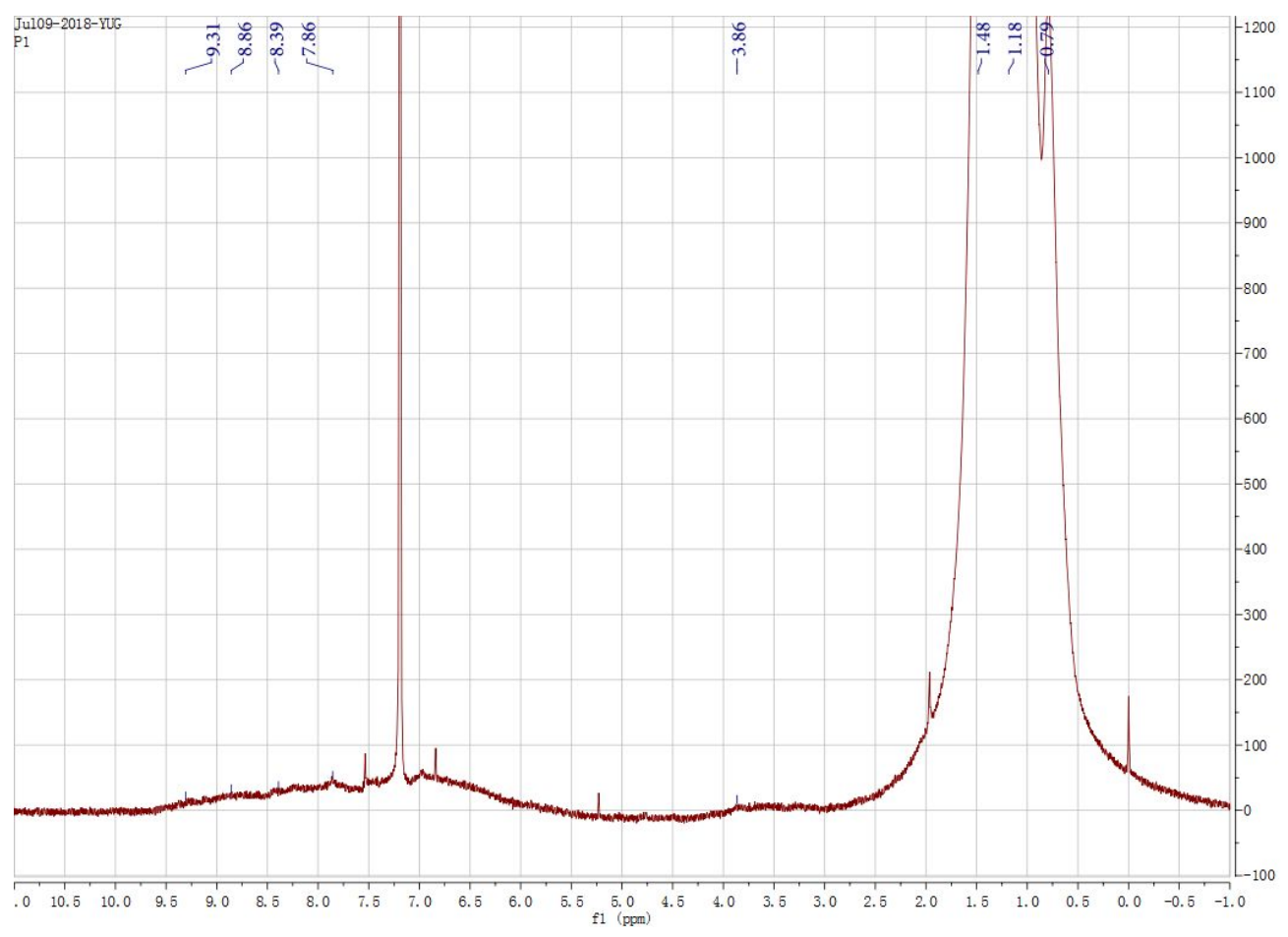

Figure S17. ${ }^{1} \mathrm{H}$ NMR spectrum of polymer PAIID-TFBVB-C1.

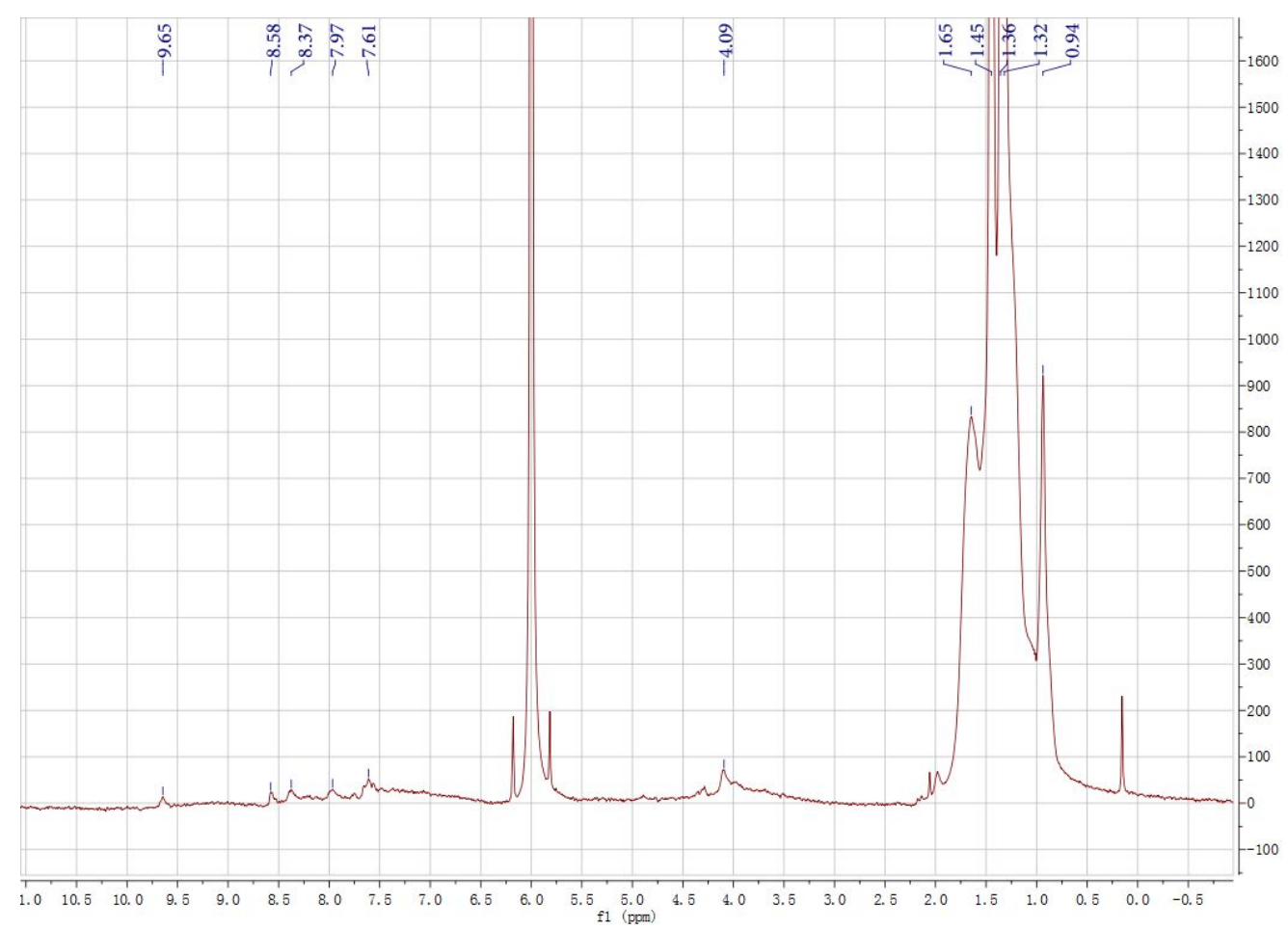

Figure S18. ${ }^{1} \mathrm{H}$ NMR spectrum of polymer PAIID-TFBVB-C3. 


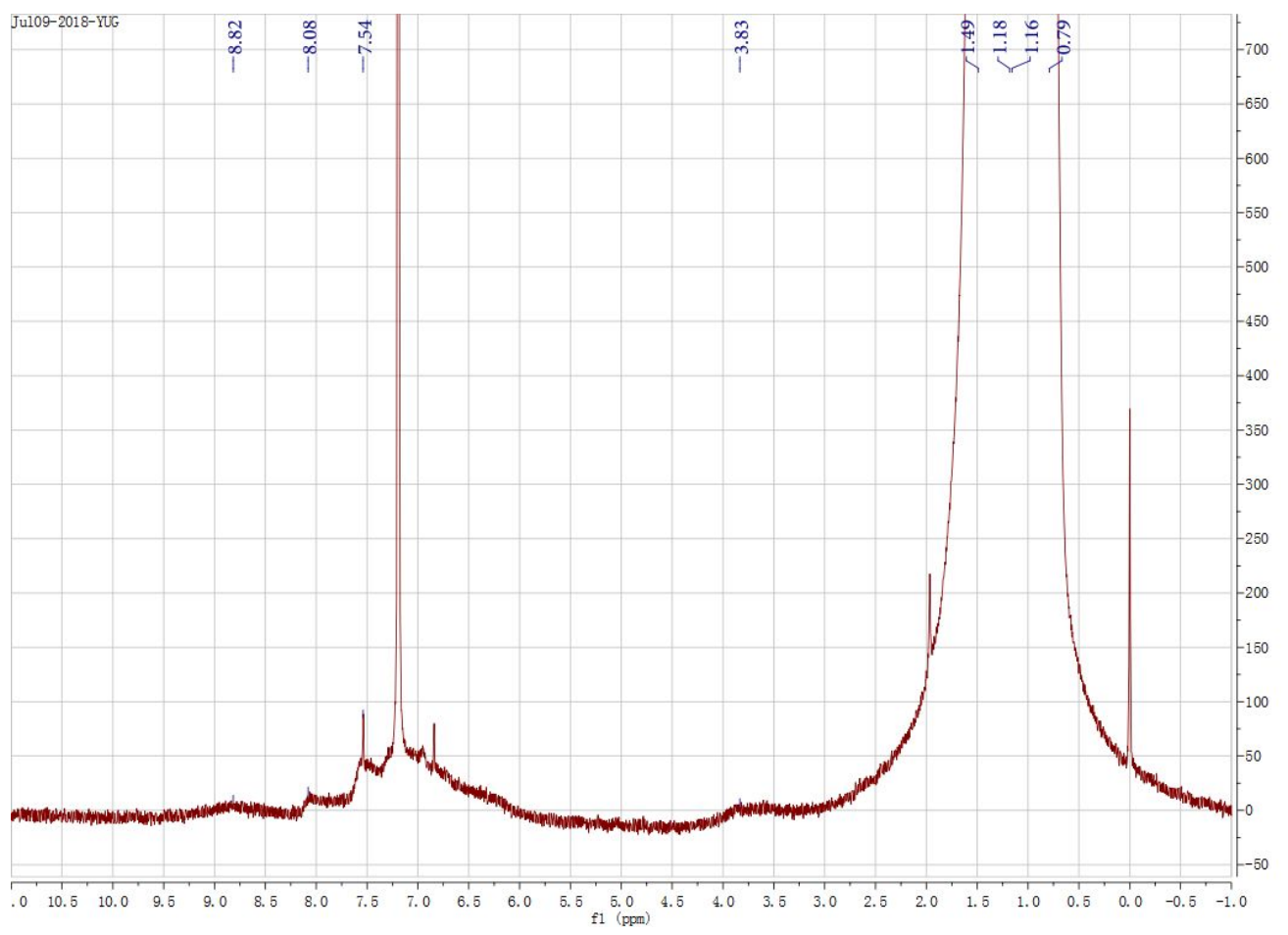

Figure S19. ${ }^{1} \mathrm{H}$ NMR spectrum of polymer PAIID-BVB-C3. 